\title{
BACK AND FORWARD BISTATIC INTERFEROMETRY
}

\author{
Sergi Duque, Paco Lopez-Dekker, Jordi J. Mallorqui and Juan C. Merlano \\ Remote Sensing Laboratory (RSLab) \\ Signal Theory and Communications Dept. (TSC) \\ Universitat Politecnica de Catalunya (UPC) \\ Email: \{sergi.duque, paco.dekker, mallorqui, juan.merlano\}@tsc.upc.edu
}

\begin{abstract}
This paper characterizes the interferometric phase for a fixedreceiver bistatic SAR system. Also, the expressions for the interferometric phase and image resolution cell are summarized. It will be distinguished between two particular acquisition geometries : back-scattering and forward-scattering. A bistatic interferometric chain has been implemented whose particularities are discussed. The theoretical developments are complemented with the comparison of the Digital Elevation Models (DEM) generated from the bistatic interferometric data acquired with our fixed receiver, named SABRINA (SAR Bistatic Receiver for INterferometric Applications) with a SRTM DEM and a Digital Terrain Model (DTM) from the Institut Cartografic de Catalunya (ICC).
\end{abstract}

Index Terms - SAR, bistatic, DEM generation

\section{INTRODUCTION}

While SAR interferometry (InSAR), differential interferometry (DInSAR) and polarimetry (Pol-SAR) are well known and mature techniques, bistatic and multistatic configurations are opening a new research front. A discussion of different bistatic and multistatic missions and experiments can be found in the literature $[1,2]$. Most of these systems can be described as quasi-monostatic, with the receiver and the transmitter close to each other in almost parallel orbits. The Remote Sensing Laboratory (RSLab) of the Universitat Politecnica de Catalunya (UPC) is studying a generic bistatic configuration in which the transmitter and receiver are far away and with large bistatic angles. A C-band receiver that uses ESA's ERS2 and ENVISAT satellites as transmitters of opportunity has been developed. This system, which has been named SABRINA (SAR Bistatic Receiver for INterferometric Applications) [3] [4], is usually placed at locations with a

This work has been supported by the Spanish MCYT and European Union FEDER funds under the projects TEC2005- 068631-C02-01 and TEC2007-65690/TCM, the Spanish Ramon y Cajal program, the Catalan Commission for Research (CIRIT) and the Spanish MEC granting the first author (AP2005-1484). DEM provided by the NASA/DLR SRTM mission and the DTM by the Institut Cartografic de Catalunya (ICC).

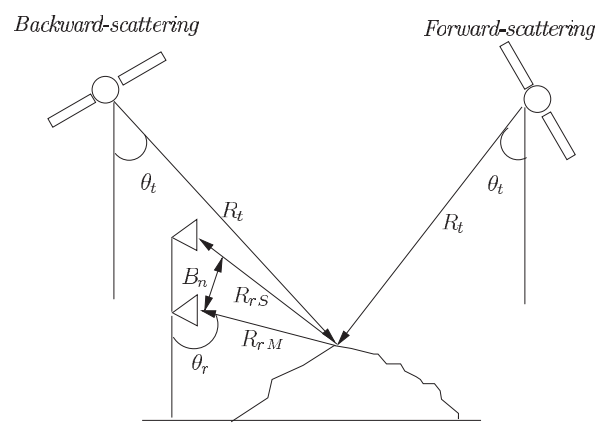

Fig. 1. Back and forward-scattering Bistatic SAR geometries for interferometric data acquisition.

good perspective of the scene, like the top of a hill or the roof of a tall building. In Section II the bistatic interferometric phase is characterized and the image bistatic resolutions presented. The particularities of the bistatic interferometric chain are highlighted in Section III. Section IV shows DEMs generated from real bistatic interferometric data for two acquisition geomeries: back and forward-scattering. The bistatic DEMs are compared with a SRTM DEM and a DTM from the ICC. Finally, Section $V$ summarizes the conclusions.

\section{BISTATIC INTERFEROMETRY}

Figure 1 illustrates the two geometric configurations for bistatic data acquisition studied in this paper. In backscattering geometries the transmitter and the receivers are on the same side of the imaged area. On the contrary, in forward-scattering geometries the imaged area is in between the transmitter and the receivers. In the figure, $\theta_{t}$ and $\theta_{r}$ are the incidence angles for the transmitter and the receiver, $\alpha$ is the local slope of the terrain and $B_{n}$ is the perpendicular baseline from master to slave antenna. $R_{r M}$ and $R_{r S}$ are the distances from target to master an slave antennas respectively. Finally, $R_{t}$ is the transmitter range. As in the monostatic case, the interferometric phase for a point is not significant by itself. Instead, the information resides in the phase difference between nearby points of the interferogram, which has the 
following general expression (removing the flat earth term):

$$
\Delta \Psi_{A B}=\frac{2 \pi}{\lambda} \cdot \Delta h_{A B} \cdot\left(\frac{B_{n}}{R_{r M} \cdot \sin \left(\theta_{r}\right)}\right)
$$

where $A$ and $B$ are two nearby points of the scene and $\Delta h_{A B}$ is the height difference between them. Notice that the interferometric phase depends only in the one-way path from scene to the antennas and $R_{t}$ is irrelevant.

An important issue about back and forward-scattering bistatic interferometric data is the image resolution. While the azimuth resolution is identical in both cases, the ground range one is significantly different. Considering a locally flat surface the ground range resolution can be approximated by [4]

$$
\Delta r_{g}=\frac{c}{\Delta f_{c} \cdot\left(\sin \left(\theta_{t}\right)+\sin \left(\theta_{r}\right)\right)},
$$

where $\Delta f_{c}$ is the transmitted signal bandwidth, and $\theta_{t}$ and $\theta_{r}$ are the local incidence angles for transmitter and receiver. If both angles are identical, 2 becomes the classical monostatic expression. In a back-scattering geometry both incidence angles have the same sign and this leads to an improvement of the resolution with respect to the orbital monostatic case. On the contrary, for forward-scattering geometries the angles have opposite signs and the resolution is worsened. Even in some cases there is a total loss of resolution when

$$
\theta_{t}=-\theta_{r}
$$

Focusing on the particular conditions of our geometry, where the receiver is close to the scene and the transmitter is onboard a satellite, 3 is only met in a small area near the receiver. Typical ground range resolution values are 13.6 meters for back-scattering configuration $\left(c=3 e 8 \mathrm{~m} / \mathrm{s}, \Delta f_{c}=16 \mathrm{MHz}\right.$, $\theta_{t}=23$ and $\left.\theta_{r}=80\right)$ and is 31.6 meters $\left(c=3 e 8 \mathrm{~m} / \mathrm{s}, \Delta f_{c}=\right.$ $16 \mathrm{MHz}, \theta_{t}=-23$ and $\theta_{r}=80$ ) for forward-scattering.

\section{BISTATIC INTERFEROMETRIC PROCESSING AND GEOCODING}

The processing that goes from bistatic raw data to a geocoded DEM starts with the focusing of the acquired data. The SAR images are generated using a back-projection algorithm [4]. After the focusing step the two resulting complex bistatic images, master and slave, are combined to generate an interferogram. Since it is assumed that a DEM is available, it makes sense to generate a synthetic interferogram and use it to cancel most of the interferometric fringes. The residual fringes will be due to the difference between the observed scene and the low resolution DEM used. Once the residual interferogram has been unwrapped the georeferenciation takes place using as input the complete unwrapped interferogram, the satellite's orbit and the precise positions of the receiver antennas. The geocoded position for each pixel can be obtained from the solution of the following system of equations:

$$
\begin{aligned}
\left(\vec{P}_{T x}-\vec{P}_{A}\right) \cdot \vec{V}_{T x} & =0 \\
\left\|\vec{P}_{R x 1}-\vec{P}_{A}\right\|+\left\|\vec{P}_{T x}-\vec{P}_{A}\right\| & =R_{b, 1} \\
\left\|\vec{P}_{R x 2}-\vec{P}_{A}\right\|-\left\|\vec{P}_{R x 1}-\vec{P}_{A}\right\| & =-\frac{\lambda}{2 \pi} \cdot \Delta \Psi_{A},
\end{aligned}
$$

where $\vec{P}_{T x}, \vec{P}_{R x 1}, \vec{P}_{R x 2}, \vec{P}_{A}$ are respectively the positions of the transmitter, the receiver master antenna and pixel $A$ in cartesian coordinates. $\vec{V}_{T x}$ is the velocity vector of the transmitter. $R_{b, 1}$ is the bistatic distance for the master receiver and $\Delta \Psi_{A}$ is the unwrapped interferometric phase for $A$. The first equation is the Doppler equation, identical that in the monostatic case as the processor locates the target on its zerodoppler azimuth position. The second equation is the bistatic range equation for the master antenna that describes an ellipsoid with one foci on the master antenna and the other on the transmitter. Finally, the third equation represents an hyperbola with the two foci at the receivers. The hyperbola defines a locus of points located where the difference of ranges from target to each antenna is constant. This range difference is obtained from the interferometric phase. The last equation can also be formulated as another ellipsoid for the slave antenna and its bistatic range calculated with the interferometric phase, as it is usually done in monostatic. But it has been proved that the hyperbola allows to solve the non-linear system of equations more efficiently.

\section{RESULTS}

This section discusses the results obtained from two experimental data sets acquired by SABRINA one for each acquisition geometry. In both cases, data was acquired using descending orbits over Barcelona. The back-scattering data was acquired using ERS-2 as a transmitter of opportunity while the forward-scattering was acquired using ENVISAT.

\subsection{Data set description}

\subsubsection{Back-scattering}

The data acquisition was on November 9th, 2007, the receivers were placed on the roof of a tall building and observing the scene under a back-scattering geometry. Figure 2 shows the set-up for the back - scattering acquisition data and the scene on the back-ground with an urban area in near range and a barely vegetated hill on far range. The two receiver antennas were separated by a vertical baseline of $77 \mathrm{~cm}$.

The maximum range was about $1 \mathrm{Km}$, which was limited by the hill in the north.

\subsubsection{Forward-scattering}

The second acquisition took place on April 16th, 2008. The system was placed in the same building but this time the an- 


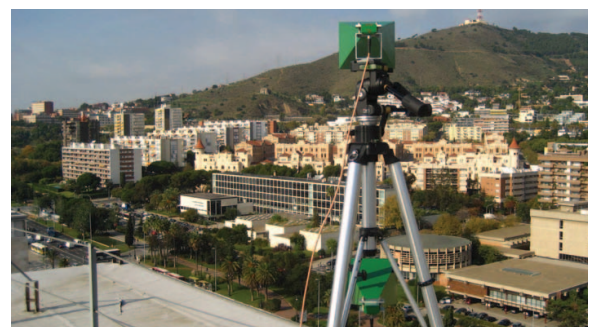

Fig. 2. Interferometric antennas and scene on the background.

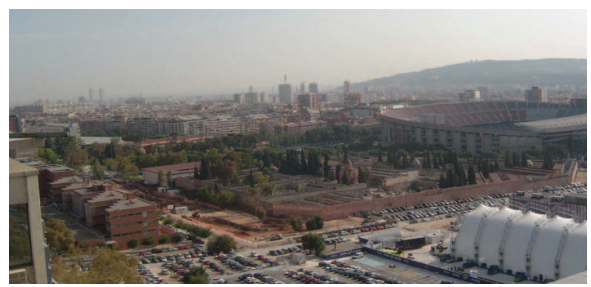

Fig. 3. Forward-scattering scene.

tennas were pointing to the opposite direction. The receiver antennas were separated $112 \mathrm{~cm}$ in vertical and the illuminated scene was larger. Most of the area is urban except a little hill in the far range near the sea, Montjuic. The scene viewed by the antennas is shown in Figure 3.

\subsubsection{DEM and error assessment}

The resulting DEM is shown in Figure 4. The DEM obtained with the back - scatering data set is located in the NorthWest while the obtained with the forward - scattered is in the South-East of the image. As said before, the forward image is larger than the back, which is limited by the hill on the North. The forward image has a lot of shadowing due that the buildings located in the antenna far range are hidden by tall ones which are at a nearer range. Also, in a forward-scattering ge-

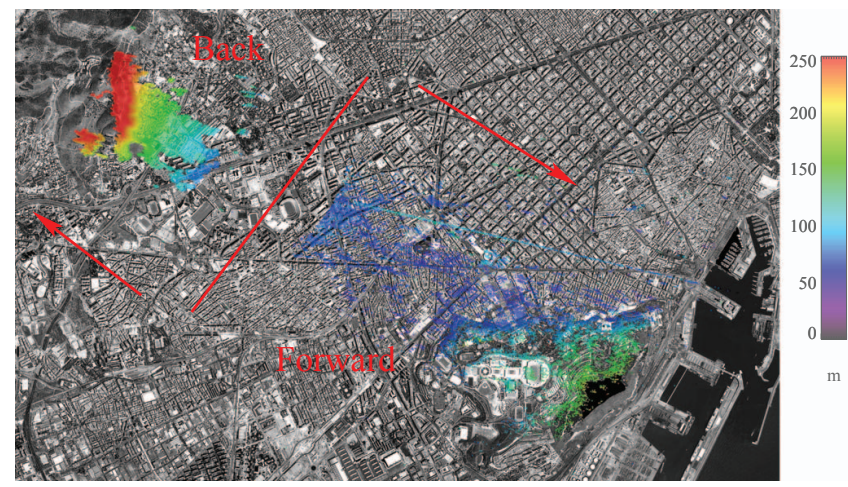

Fig. 4. DEM from the back-scattering experiment.

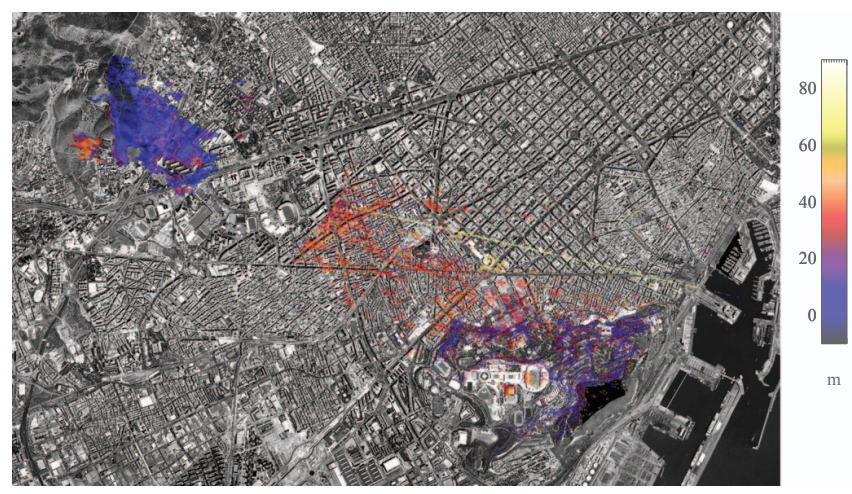

Fig. 5. Height difference between DEM produced using bistatic data and the DTM

ometry the double shadow effect inherent to bistatic radar is maximized, while in a back-scattering is more similar to the monostatic case. The calculated DEM clearly matches the underlaying geometry, revealing the Sant Pere Martir Hill at the North-West and Montjuic at the south-East. In order to evaluate the DEM with more detail and to carry out an error assessment, it has been compared with a reference Digital Terrain Model (DTM, where the heights of the buildings are not included) provided by the ICC. The difference between our DEM and the DTM is shown in Figure 5.

Intuitively, it is possible to observe that in the urban area the difference is higher and positive while in the hill near the sea and in the hill in the north the difference is close to zero. This is consistent with the near absence of buildings on the hill slopes. Therefore, we can conclude that the height difference is due to the buildings. Figure 6 shows a detail of this height difference around the St Jordi stadium.Figure 6(a)

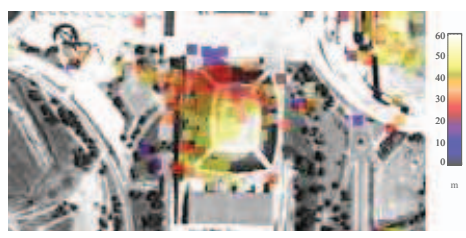

(a)

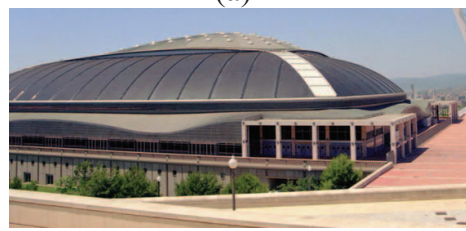

(b)

Fig. 6. (a) Retrieved height of the St Jordi Stadium; (b) Photo of the stadium's dome.

shows how the shape of the St Jordi's dome, which can be seen in the photo of Figure 6(b), is retrieved correctly. A 


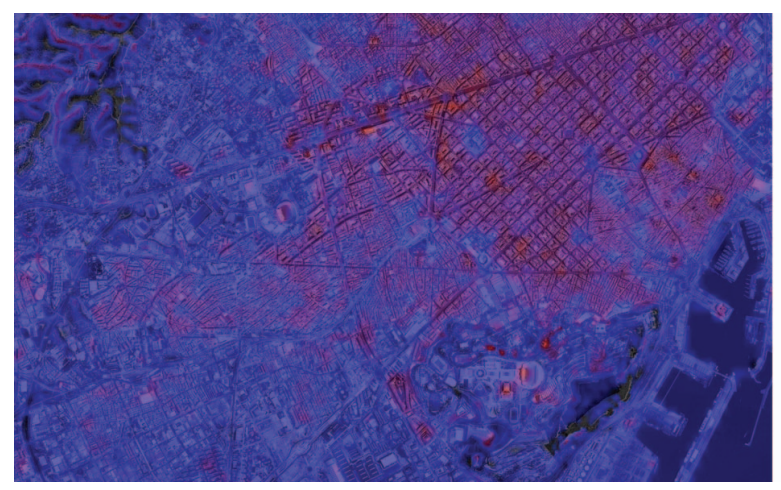

Fig. 7. Height difference between SRTM DEM and the DTM

SRTM DEM of the scene have been also compared with the DTM, the height difference between them is shown in Figure 7.

The height difference in this case is smaller, this is due two reasons. Firstly, the looking angle of the SRTM is less than the bistatic receiver. Thus, it is possible to look the streets, which have no height with respect to the DTM, while the bistatic receiver is looking to the roof of the buildings. The second reason is the resolution of each DEM, the SRTM used has a resolution cell of $90 \times 90 \mathrm{~m}$, which implies and avering of all the scatterers in the resolution cell, while the bistatic DEM has a better resolution cell and then less avering.

To compare the height differences of the DEMs with respect to the DTM, its probability density function has been calculated for the selected pixels in the bistatic data. Figure 8(a) shows the height differences $p d f$ s with respect to the DTM in the back-scattering scenario for the SRTM DEM (a) and the bistatic DEM (a).

Both $p d f$ s are quite similar, however, the bistatic DEM one is wider and shows a positive bias with respect to the SRTM DEM. The bias is due to the looking angle, the bistatic receiver mostly sees buildings while SRTM both buildings and streets. The bistatic $p d f$ is wider because the improved resolution. This improved resolution decreases the implicit averaging of heights

The same $p d f$ analysis has been for the forward-scattering data, the results are shown in Figure 8(b).Again, there is a bias with respect to the SRTM pdf. Moreover, in this data the bistatic $p d f$ is much wider than in the back-scattering data. It can be explained by the nature of the observed scene, the forwad-scattering scenario is much more urban and, therefore, more heterogeneous.

\section{CONCLUSIONS}

The DEM generation using bistatic interferometric data has been validated. It has been shown that the DEM obtained us-

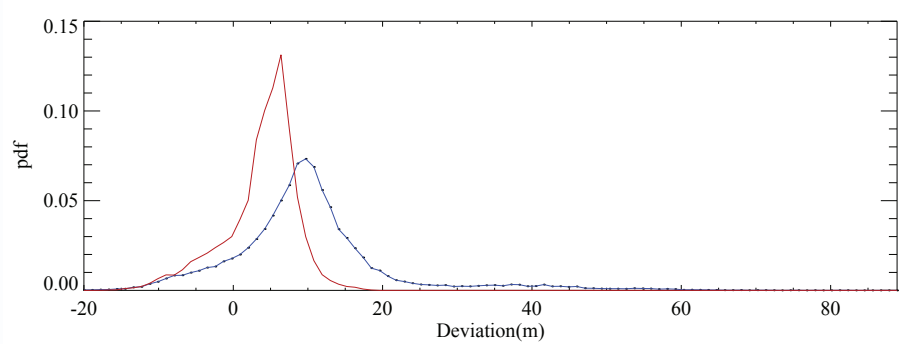

(a)

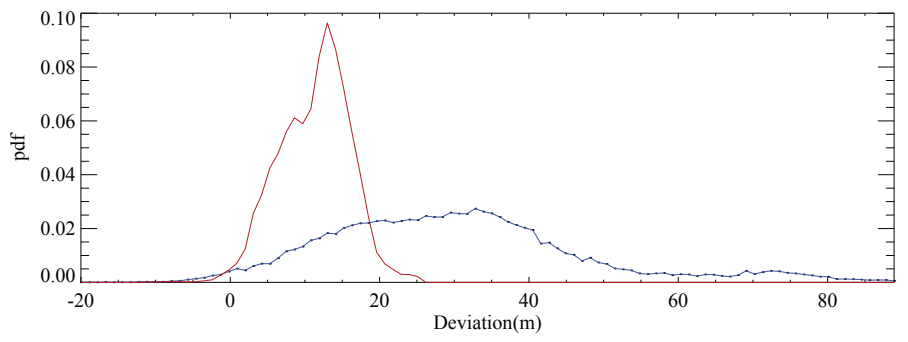

(b)

Fig. 8. Height differences histograms with respect to the DTM in the forward-scattering scene for SRTM DEM(red) and for bistatic DEM(blue) and for back-scattering scenario (a) and forward-scattering (b).

ing bistatic interferometric data is comparable with an SRTM DEM taking into account the looking angles and the poor resolution of the SRTM. It has to be highlighted that the final purpose of SABRINA is to produce differential bistatic applications. Thus, the bistatic DEM generation is a required step in the development of bistatic interferometric applications.

\section{REFERENCES}

[1] M. Zink, H. Fiedler, I. Hajnsek, G. Krieger, A. Moreira, and M. Werner. The tanDEM-X mission concept. In Proceeding of International Geoscience and Remote Sensing Symposium, 2006.

[2] I. Walterscheid, J.H. Ender, A. R. Brenner, and O. Loffeld. Bistatic SAR processing and experiments. IEEE Transactions on Geoscience and Remote Sensing, 44(10):2710-2717, 2006

[3] J Sanz-Marcos, P. Prats, J Mallorqui, and A. Aguasca. A subaperture range-doppler processor for bistatic-fixedreceiver SAR. In EUSAR06, 2006.

[4] J. Sanz-Marcos, P. Lopez-Dekker, J. J. Mallorqui, A. Aguasca, and P. Prats. SABRINA: a SAR bistatic receiver for interferometric applications. IEEE Geoscience and Remote Sensing Letters, Vol 4:pages 307-311, 2007. 